\title{
The Long Intergenic Noncoding RNA 00707 Promotes Lung Adenocarcinoma Cell Proliferation and Migration by Regulating Cdc42
}

\author{
Tianshi Maa Hongwei Ma ${ }^{b}$ Zigui Zou ${ }^{a} \quad$ Xuezhi Hec Yanhua Liud You Shuaie \\ Min Xie ${ }^{f}$ Zhihong Zhanga \\ aDepartment of Pathology, The First Affiliated Hospital of Nanjing Medical University, Nanjing, \\ 'Department of Pathology, Zhenjiang First people's hospital, Zhenjiang, 'Department of Anatomy, \\ Histology and Embryology, Nanjing Medical University, Nanjing, dDepartment of Oncology, Xuzhou \\ Central Hospital, Affiliated Xuzhou Hospital, College of Medicine, Southeast University, Xuzhou, \\ eDepartment of Oncology, The First Affiliated Hospital of Nanjing Medical University, Nanjing, \\ ${ }^{f}$ Central Laboratory, The Affiliated Suzhou Hospital of Nanjing Medical University, Suzhou Municipal \\ Hospital, Suzhou, Jiangsu, People's Republic of China
}

\section{Key Words}

Lncrna $•$ LINC00707 • LAD • Cdc42 • Proliferation • Migration

\begin{abstract}
Background/Aims: Lung cancer (LC) is a serious disease with high morbidity and mortality. Long noncoding RNAs (IncRNAs) have garnered attention because they participate in diverse human disorders, including cancer. Our study examined the long intergenic noncoding RNA 00707 (LINC00707). The effects of LINC00707 on lung adenocarcinoma (LAD) and molecular mechanisms are unclear. This study is aimed to investigate the role of LINC00707 in the malignant processes of LAD. Methods: Quantitative reverse transcription PCR (qRT-PCR) was used to examine the expression level of LINC00707 in tissues and cell lines. The association of LINC00707 expression and postoperative prognosis was analyzed by the Kaplan-Meier method and log-rank test. Cell proliferation was evaluated in vitro and in vivo. Transwell assays were performed to examine cell migration. Cell cycle and apoptosis was determined by flow -cytometric and western blot analyses. Microarray analysis was conducted to screen for the downstream target gene Cdc42 of LINC00707, which was identified by qRT-PCR, functional analysis, and rescue experiment. Results: The expression level of LINC00707 was clearly upregulated in LAD tissues compared to that in corresponding normal tissues. Its overexpression was related to advanced TNM stage, larger tumor size, lymphatic metastasis, and poor prognosis. Functional assays revealed that LINC00707 knockdown repressed LAD cell proliferation both in vitro and in vivo. This process may involve the inducing of G1 arrest and apoptosis. Moreover, cell migration was impaired after LINC00707 inhibition. Microarray analysis and rescue assays suggested that $\mathrm{Cdc} 42$ is an important target gene involved in the

T. Ma, H. Ma and Z. Zou contributed equally to this work.

Zhihong Zhang and Min Xie

Department of Pathology, The First Affiliated Hospital of Nanjing Medical University,

300 Guangzhou Road, Nanjing (People's Republic of China)

E-Mail zhangzh@njmu.edu.cn, xmszsl90@163.com
\end{abstract}

KARGER 
carcinogenesis of LINC00707. Conclusions: In summary, LINC00707 is a noncoding oncogene that exerts important regulatory functions in $L A D$, suggesting its potential as a biomarker in the prognosis and treatment of LAD.

\section{Introduction}

Lung cancer (LC) is the leading cause of cancer-related death among males and second leading cause among females [1]. Non-small cell lung cancer (NSCLC) currently accounts for approximately $80 \%$ of all lung cancer cases [2], with adenocarcinoma being the most frequently encountered histology within NSCLC [3]. Despite comprehensive approaches including chemotherapy, radiation treatment, surgical operation, and molecular targeted therapy which are implemented in the clinic, the survival rate of advanced lung adenocarcinoma (LAD) patients remains low $[4,5]$. Thus, the pathogenesis mechanisms of LAD should be examined. Currently, dozens of molecular pathways and mechanisms have been confirmed to affect tumor oncogenesis and progression [6, 7]. However, the molecular biological mechanisms and biomarkers involved in LAD remain unclear.

With the advent of high-resolution microarray and whole-genome sequencing technology, it was found that only $2 \%$ of the human genome encodes for proteins, while the remainder contains noncoding genes [8-10]. As an increased number of functions for noncoding RNAs (ncRNAs) are being indentified, short ncRNAs (<200 nt) and long ncRNAs (>200 nt) have gained attention $[9,11]$. Among them, lncRNAs can regulate transcription networks at multiple levels through their limited protein coding ability [12]. Although lncRNAs were previously regarded as "transcriptional noise", they are now considered as key regulators of physiological processes and disorders including cancer [11, 13]. For instance, lncRNA H19 functions as an essential mediator of bone morphogenetic protein 9-induced osteogenesis differentiation of mesenchymal stem cells through Notch signaling, and dysregulation of H19 expression may impair normal osteogenesis [14]. Magistri et al. showed using next generation RNA sequencing that several IncRNAs are differentially expressed in late-onset Alzheimer's disease brain tissues [15]. Particularly, a relationship between IncRNA and LAD has been suggested. LncRNA LINC00152 is upregulated remarkably in LAD, which promotes cell proliferation by binding to enhancer of zeste homology 2 and repressing the expression of interleukin 24 [16]. LncRNA CCAT1 is responsible for the chemoresistance of docetaxelresistant LAD cells through sponging let-7c and releasing Bcl-xl [17]. LncRNA XIST promotes cisplatin resistance of LAD cells via competing with and repressing let-7i, which regulates its target BAG-1 [18]. Therefore, IncRNAs are vital factors in oncogenesis, but the comprehensive pathophysiological contributions of IncRNAs to LAD are unclear.

Long intergenic noncoding RNA 00707 (LINC00707), a 3087bp ncRNA, located on chromosome 10p14, has unknown biological functions in LAD. In this study, we found that LINC00707 was significantly upregulated in LAD compared to that in adjacent normal tissues. Moreover, upregulation of LINC00707 was correlated with several clinical indicies, such as advanced TNM stage, lymphatic metastasis, larger tumor size, and poor prognosis. Further assays revealed that LINC00707 promoted cell proliferation and migration by regulating its target gene Cdc42. These results indicated that LINC00707 played a noncoding oncogenic role in neoplasia and functions as a latent biomarker for the diagnosis and therapy of LAD.

\section{Materials and Methods}

Tissue samples and clinical data collection

A total of 64 paired LAD and corresponding nontumorous lung tissues were obtained from patients who underwent surgery at First Affiliated Hospital of Nanjing Medical University. No local or systemic treatment had been implemented on these patients before surgery. All cases were verified as LAD based on histopathological evaluation. The clinicopathological characteristics of the LAD patients are summarized in Table 


\section{Cellular Physiology Cell Physiol Biochem 2018:45:1566-1580

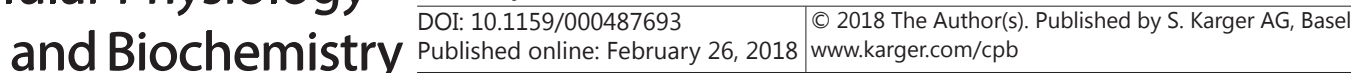 \\ Ma et al.: Oncogenic Functions of LINC00707 in LAD}

1. All the collected samples were immediately snap-frozen in liquid nitrogen and stored at $-80^{\circ} \mathrm{C}$ until required. Our study was in accordance with the Declaration of Helsinki and approved by the Research Ethics Committee of Nanjing Medical University, China. Written informed consent was obtained from all patients.

\section{Cell culture}

Four human LAD cell lines (SPCA1, A549, H1299, H1975) and the normal human bronchial epithelial cell line (16HBE) were purchased from the Institute of Biochemistry and Cell Biology of the Chinese Academy of Sciences (Shanghai, China). SPCA1 and 16HBE cells were cultured in Dulbecco's Modified Eagle Medium (DMEM; GIBCOBRL); A549 cells were grown in RPMI-1640 medium (GIBCO-BRL) supplemented with 10\% fetal bovine serum (FBS; ScienCell) and antibiotics (100 U/ml penicillin and 100 $\mathrm{mg} / \mathrm{ml}$ streptomycin) (Invitrogen, Carlsbad, CA, USA) in humidified air at $37^{\circ} \mathrm{C}$ with $5 \% \mathrm{CO}_{2}$.

\section{RNA isolation and qRT-PCR analyses}

Total RNA was extracted from tissues or cultured cells using TRIZOL reagent (Invitrogen). RNA ( $1 \mu \mathrm{g})$ was reverse transcribed to cDNA in a final volume of $20 \mu \mathrm{l}$ under standard conditions with the PrimeScript RT Reagent Kit (Takara, Dalian, China). Real-time PCR analyses were performed with SYBR Premix Ex Taq (Takara) in accordance with the manufacturer's protocol. Results were normalized to the expression of glyceraldehyde 3-phosphate dehydrogenase (GAPDH), and data were analyzed by comparative cycle

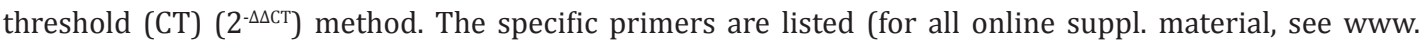
karger.com/doi/10.1159/000487693) in Table S1.

\section{RNA interference}

SPCA1 and A549 cells were transfected with siRNAs using Lipofectamine 2000 (Invitrogen, USA) according to the manufacturer's instructions. Three individual LINC00707 siRNAs (si-LINC00707 1\#, 2\#, 3\#), Cdc42 siRNA and scrambled negative control siRNA (si-NC) were purchased from Invitrogen. The nucleotide sequences of siRNAs for LINC00707, Cdc42 are listed (see online suppl. material) in Table S1. Cells were harvested for qRT-PCR or western blot analysis $48 \mathrm{~h}$ after transfection.

\section{Plasmid generation}

SPCA1 and A549 cells were transfected with plasmid vector using XtremeGENE HP DNA transfection reagent (Roche, Basel, Switzerland) according to the manufacturer's instructions. The full-length complementary DNA of LINC00707 was synthesized by Realgene (Nanjing, China) and subcloned into the pcDNA3.1(+) vector (Invitrogen). At transfection for $48 \mathrm{~h}$, cells were harvested for qRT-PCR or western blot analysis.

Cell proliferation assays

Cell viability was detected with Cell Proliferation Reagent Kit I (MTT; Roche Applied Science). SPCA1 and A549 cells transfected with si-LINC00707 or pcDNA-LINC00707 were grown on 96-well plates (3000 


\section{Cellular Physiology Cell Physiol Biochem 2018;45:1566-1580

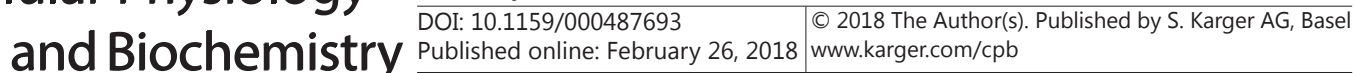 \\ Ma et al.: Oncogenic Functions of LINC00707 in LAD}

cells/well). Cell viability was measured every $24 \mathrm{~h}$ following the manufacture's protocol. All experiments were performed in triplicate. For the colony formation assay, a certain number of transfected cells were seeded in each well of six-well plates and maintained in appropriate medium containing $10 \%$ fetal bovine serum for 14 days, during which the medium was replaced every 4 days. After 2 weeks, the cells were treated with methanol to fix and stained with $0.1 \%$ crystal violet (Sigma-Aldrich). Visible colonies were then counted. For each treatment group, wells were performed in triplicate, and experiments were independently repeated three times.

\section{Cell Migration Assay}

Transfected cells were seeded in 24-well Transwell chambers with an 8-mm pore size polycarbonate membrane (Corning Incorporated). After $24 \mathrm{~h}$ incubation, cells inside the upper chamber were wiped with cotton swabs, whereas cells on the lower membrane surface were fixed with methanol and then stained with $0.5 \%$ Crystal violet solution. Five randomly selected fields were counted in each well.

\section{Ethynyldeoxyuridine (EdU) analysis}

Proliferating cells were assessed using an EdU labeling/detection kit (Ribobio, Guangzhou, China) basing on the manufacturer's protocol. Cells were grown in 96 -well plates at $5 \times 10^{3}$ cells per well. $48 \mathrm{~h}$ after transfection, $50 \mu \mathrm{M}$ EdU labeling medium was added to cell culture and they were incubated for $2 \mathrm{~h}$ at $37^{\circ} \mathrm{C}$ under 5\% CO2. Next, the cultured cells were treated with $4 \%$ paraformaldehyde (pH 7.4 ) and then $0.5 \%$ Triton X-100 for $30 \mathrm{~min}$ and $20 \mathrm{~min}$ respectively at room temperature. Then the cells were stained with antiEdU working solution and subsequently incubated with $100 \mu \mathrm{l}$ Hoechst $33342(5 \mu \mathrm{g} / \mathrm{ml})$. The percentage of EdU-positive cells was observed under fluorescent microscopy. The percentage of EdU-positive cells was calculated from five random fields in three wells.

\section{Flow cytometric analysis}

SPCA1 and A549 cells transfected with si-LINC00707 or scrambled were harvested $48 \mathrm{~h}$ after transfection by trypsinisation. Using the FITC Annexin V Apoptosis Detection Kit (BD Biosciences), double staining with FITC-Annexin V and propidium iodide (PI) had been performed following the protocol. Then the cells were analyzed by flow cytometry (FACScan $\AA$; BD Biosciences) with CellQuest software (BD Biosciences). Cells were classified into viable, dead, early apoptotic, and apoptotic cells, and the ratio of early apoptotic cells was compared with the control for each experiment. Cells for cell cycle analysis were stained with PI using the AnnexinV Alexa Fluor647/PI/Apoptosis detection kit (Fcmacs, China) in accordance with the manufacturer's recommendations, and analyzed by FACScan. The percentage of cells in G0/G1, S, and G2/M phase were counted respectively and compared.

\section{Western blot assay and antibodies}

Cells protein lysates separated by 10\% SDS-polyacrylamide gel electrophoresis (SDS-PAGE) were transferred to $0.22 \mu \mathrm{m} \mathrm{NC} \mathrm{membranes} \mathrm{(Sigma)} \mathrm{and} \mathrm{incubated} \mathrm{with} \mathrm{specific} \mathrm{antibodies.} \mathrm{The} \mathrm{ECL} \mathrm{chromogenic}$ substrate was used to quantify by densitometry (Quantity One software; Bio-Rad). A GAPDH antibody was used as a control, and CyclinD3, CyclinD1, CDK6, CDK4, CDK2 (1:1000) were purchased from Cell Signaling Technology, Inc (CST).

\section{Tumor formation assay in a nude mouse model}

Male BALB/c-nude mice (4-weeks-old) were maintained under specific pathogen-free (SPF) conditions and manipulated according to protocols approved by the Shanghai Medical Experimental Animal Care Commission. A549 cells were stably transfected with sh-LINC00707 and empty vector and harvested from 6-well cell culture plates, washed with phosphate-buffered saline (PBS), and re-suspended at a concentration of $1 \times 10^{8}$ cells $/ \mathrm{ml}$. A total of $100 \mu \mathrm{l}$ of suspended cells was subcutaneously injected into a single side of the armpit of each mouse. Tumor growth was examined every 5 days, and tumor volumes were calculated using the equation $\mathrm{V}=0.5 \times \mathrm{D} \times \mathrm{d}^{2}(\mathrm{~V}$ represents volume; $\mathrm{D}$ represents longitudinal diameter; $\mathrm{d}$ represents latitudinal diameter). At 28 days post-injection, mice were euthanized, and the subcutaneous growth of each tumor was examined. This study was performed strictly in accordance with the recommendations in the Guide for the Care and Use of Laboratory Animals of the National Institutes of Health. The protocol was approved by the Animal Ethical and Welfare Committee of Nanjing Medical University. 


\section{Cellular Physiology Cell Physiol Biochem 2018;45:1566-1580 \begin{tabular}{ll|l} 
and Biochemistry Published onlIne: February 26, 2018 & $\begin{array}{l}\text { (c) } 2018 \text { The Author(s). Published by S. Karger AG, Basel } \\
\text { www.karger.com/cpb }\end{array}$ \\
\hline
\end{tabular}

Subcellular fractionation location

The separation of nuclear and cytosolic fractions was performed using the PARIS Kit (Life Technologies, Carlsbad, CA) according to the manufacturer's protocol.

Immunohistochemical (IHC) analysis

The primary tumors were immunostained for Ki-67 as previously depicted [19].

\section{Statistical analysis}

The Students t-test (two-tailed), one-way ANOVA, and the Mann-Whitney U test were conducted to analyze in vitro and in vivo data by SPSS 17.0 software. P values less than 0.05 were recognized as significant.

\section{Results}

\section{LINC00707 is overexpressed in human LAD tissues}

We first analyzed the expression level of LINC00707 in 57 pairs of human LAD tissues using the bioinformatics tool "TANRIC" (http://ibl.mdanderson.org/tanric/_design/basic/ index.html) downloaded from The Cancer Genome Atlas database, and found that LINC00707 was remarkably overexpressed in LAD tissues compared to that in adjacent normal tissues (Fig. 1A). Moreover, qRT-PCR analysis showed that the expression of LINC00707 was increased in 64 LAD tumor tissues compared to that in paired noncancerous samples (Fig. 1B). These results suggest that upregulation of LINC00707 is important in LAD development.

LINC00707 expression correlates with TNM stage, tumor size, lymphatic metastasis and poor prognosis of LAD patients

To further investigate the importance of LINC00707 upregulation in LAD, we evaluated the association between LINC00707 expression and clinicopathological characteristics. The 64 LAD patients were divided into two groups according to the relative LINC00707 expression with median ratio: relatively high LINC00707 group ( $\mathrm{n}=32$, LINC00707 expression ratio $\geq$ median ratio) and relatively low LINC00707 group ( $n=32$, LINC00707 expression ratio $\leq$ median ratio) (Fig. 1B). Table 1 shows the clinicopathological features of the 64 LAD patients. High LINC00707 expression in LAD was significantly related to advanced TNM stage $(\mathrm{p}=0.024)$, lymphatic metastasis $(\mathrm{p}=0.019)$, and larger tumor size $(\mathrm{p}=0.031)$. However, age $(p=0.133)$, gender $(p=0.448)$, or smoking history $(p=0.209)$ were not correlated with LINC00707 expression in LAD.

Fig. 1. Relative expression of LINC00707 in LAD tissues and its clinical significance. (A)Data collected from TCGA database showed relative expression of LINC00707 in LAD tissues $(n=57)$ and their corresponding nontumorous tissues ( $\mathrm{n}=57)$. (B) Relative LINC00707 expression in LAD tissues $(n=64)$ compared with their adjacent non-cancerous tissues analyzed by qRTPCR and tissues were divided into two groups according to the fold-change of LINC00707 expression. (C and D) Kaplan-Meier progression-free survival and overall survival curves according to LINC00707 expression levels. Error bars indicate mean \pm standard errors of the mean. $* \mathrm{P}<0.05,{ }^{* *} \mathrm{P}<0.01$.

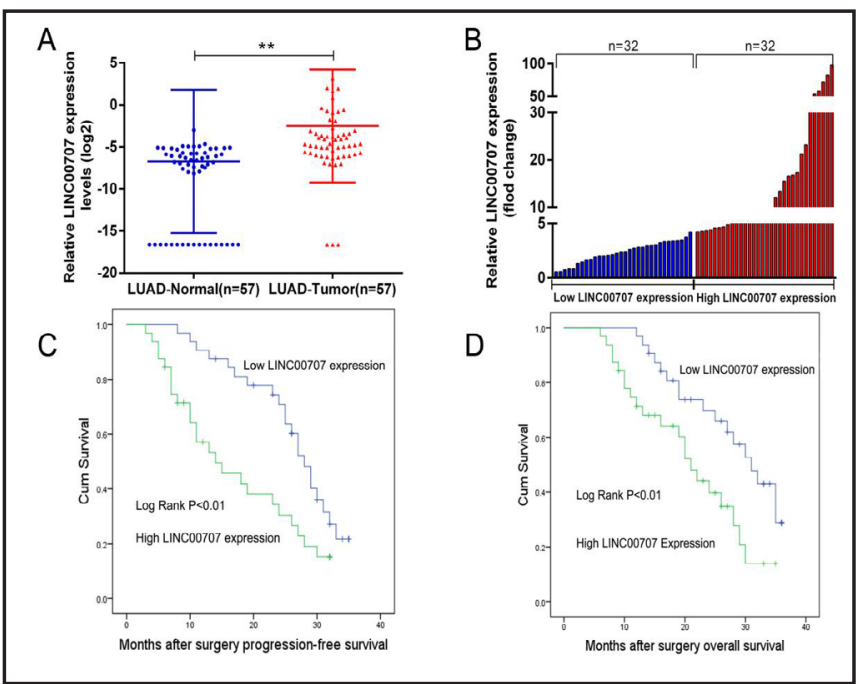




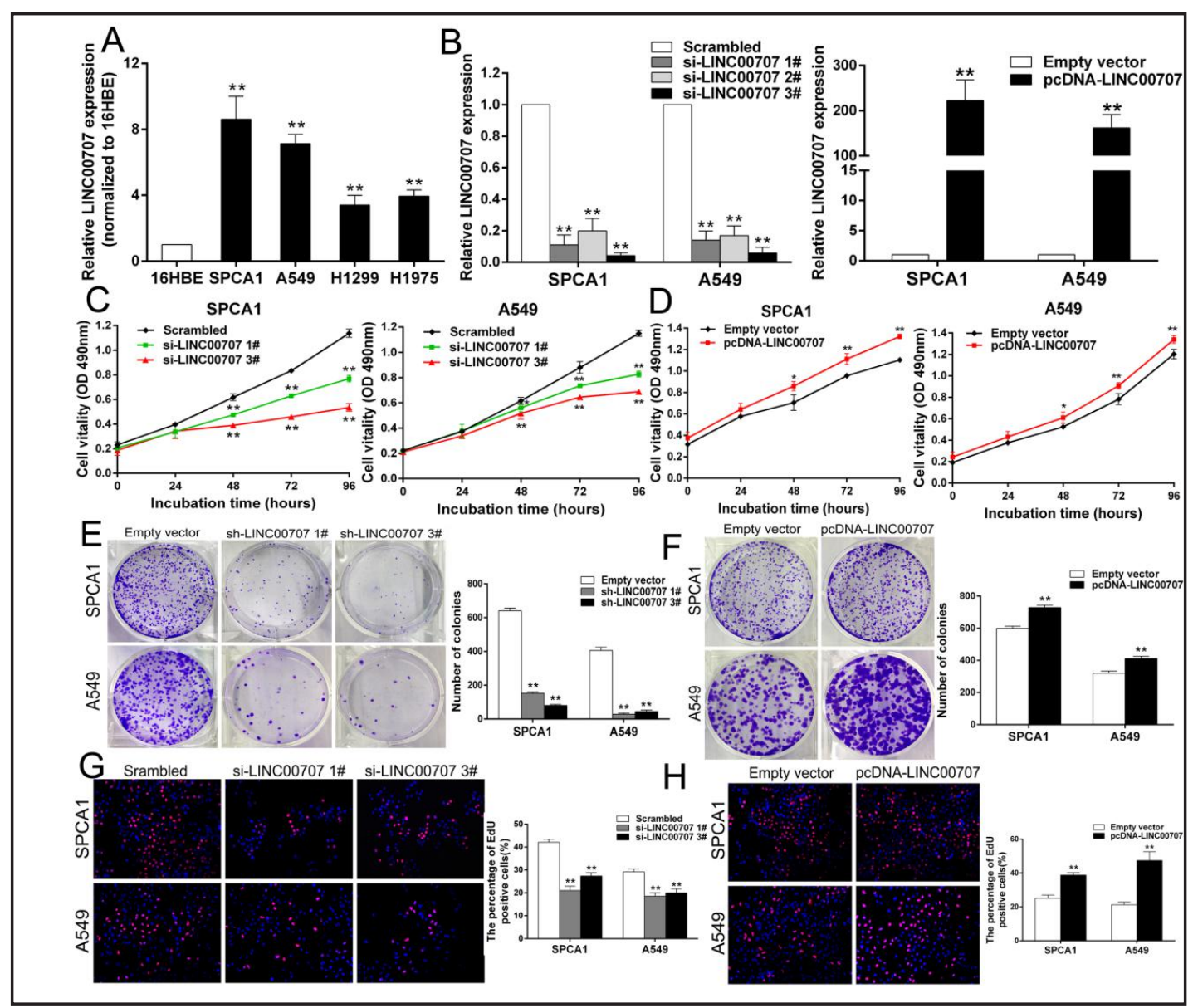

Fig. 2. LINC00707 promotes LAD cell proliferation in vitro. (A) QRT-PCR assay examined LINC00707 expression in normal bronchial epithelial cell line (16HBE) and LAD cell lines. (B) Left: QRT-PCR analysis of LINC00707 expression in LAD cells transfected with control (scrambled), si-LINC00707 1\#, si-LINC00707 2\# and si-LINC00707 3\#. Right: Relative expression of LINC00707 in LAD cells transfected with empty vector and pcDNA-LINC00707. (C and D) MTT assays were performed to determine the viability of LAD cells treated with si-LINC00707 or pcDNA-LINC00707. (E and F) Colony formation assays were used to detect the proliferation of sh-LINC00707-transfected or pcDNA-LINC00707-transfected LAD cells. Colonies were counted and captured. ( $G$ and $H$ ) Proliferous LAD cells were displayed by EdU immunostaining assays. EdU positive cells were counted and captured. Values are shown as the mean \pm s.d in three independent experiments. ${ }^{*} \mathrm{P}<0.05,{ }^{* *} \mathrm{P}<0.01$.

Progression-free survival (PFS) and overall survival (OS) curves were used to assess the relationship between the expression level of LINC00707 and postoperative prognosis and analyzed by the Kaplan-Meier method and log-rank test. Notably, patients expressing higher levels of LINC00707 had poorer disease-free survival ( $p=0.008)$ and overall survival $(\mathrm{p}=0.01)$ (Fig. 1C and D). These results indicate that the LINC00707 expression level is a valuable indicator of prognosis and progression in LAD.

\section{LINC00707 promotes LAD cell proliferation in vitro}

To explore the function of LINC00707 in LAD cells, qRT-PCR analysis was performed to detect its expression level in diverse human LAD cell lines. Fig. 2A shows that SPCA1 and A549 cells expressed higher levels of LINC00707 than other LAD cell lines and a normal bronchial epithelial cell line (16HBE). Next, we synthesized three small interfering RNAs (siRNAs) to silence LINC00707 expression and then conducted qRT-PCR analysis after 48h of transfection. The data showed that all LINC00707 siRNAs entered the cells effectively. 
Furthermore, si-LINC00707 1\# and 3\# showed more efficient interference than si-LINC00707 2\# (Fig. 2B). Thus, si-LINC00707 1\# and 3\# were analyzed in subsequent experiments. Because of the highest interference efficiency of si-LINC00707 3\#, we designed a short hairpin RNA (shRNA) according to the sequence of the siRNA for stable transfection (see online suppl. material, Fig. S1A). We also upregulated LINC00707 expression by transfecting SPCA1 and A549 cells with a pcDNA-LINC00707 expression vector. QRT-PCR revealed that the expression of LINC00707 was increased by 222- and 161- fold in SPCA1 and A549 cells, respectively, compared to that in cells transfected with an empty vector (Fig. 2B).

Because lncRNAs participate in many biological processes, we investigated the influence of LINC 00707 on LAD in vitro. MTT assays revealed that the cell viability of SPCA1 and A549 cells was significantly inhibited after impairing LINC00707 expression compared to that in control cells (Fig. 2C). In contrast, cells treated with pcDNA-LINC00707 showed higher cell viability (Fig. 2D). In addition, the results of colony formation assays showed that clonogenic survival was greatly attenuated following knockdown of LINC00707 in SPCA1 and A549

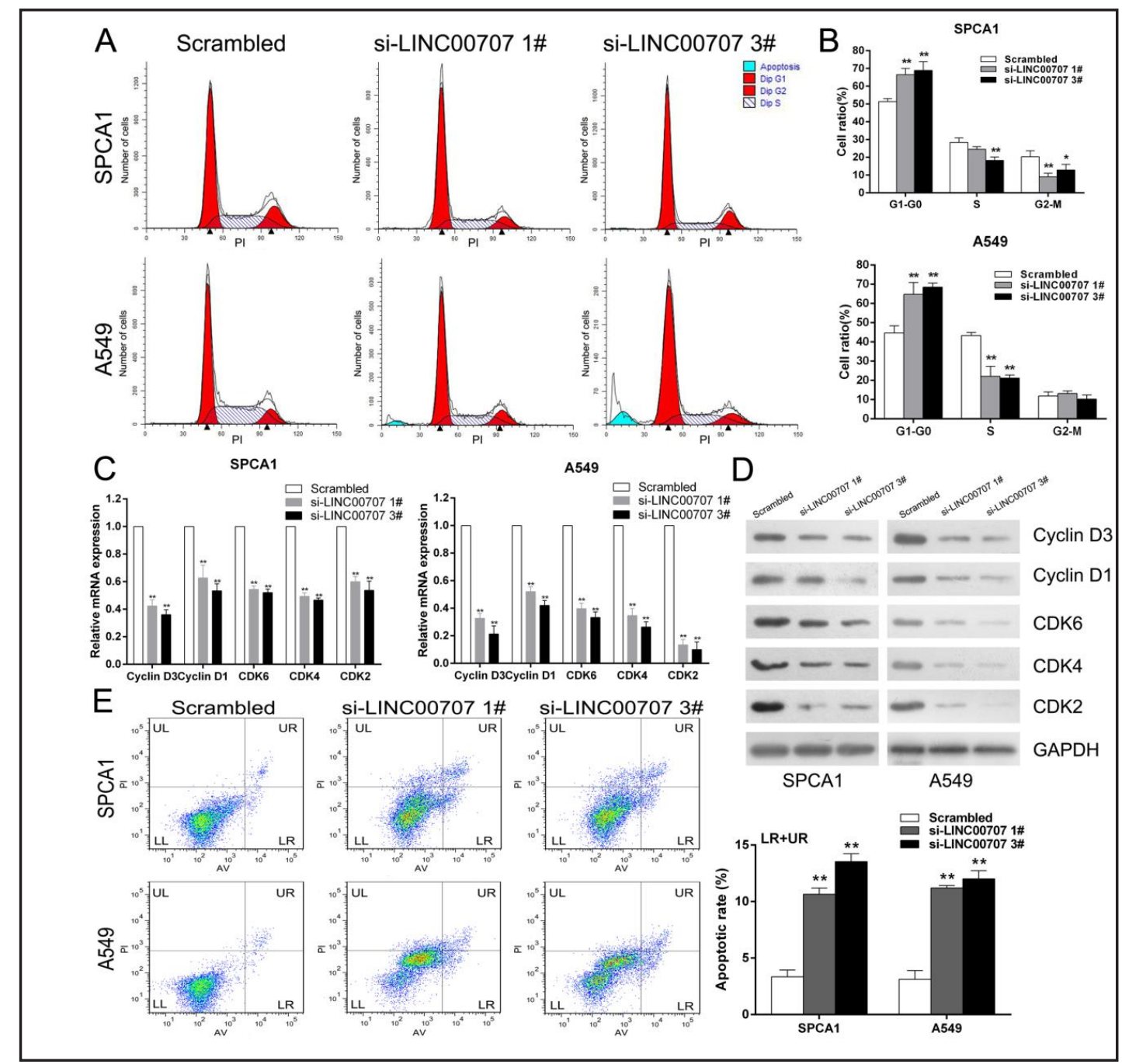

Fig. 3. Inhibition of LINC00707 induces G1 arrest apoptosis in LAD cells. SPCA1 and A549 cells were transfected with si-LINC00707 1\#, 3\# or scrambled. (A and B) Cell cycle was determined in LAD cells. (C) QRT-PCR analysis of CyclinD3, CyclinD1, CDK6, CDK4, CDK2 in LAD cells. (D) Western blot analysis of CyclinD3, CyclinD1, CDK6, CDK4, CDK2 in LAD cells. GAPDH protein was used as an internal control. (E) Apoptotic rates of cells were tested by flow cytometry. Values are shown as the mean \pm s.d in three independent experiments. ${ }^{*} \mathrm{P}<0.05$, ${ }^{* *} \mathrm{P}<0.01$. 


\section{Cellular Physiology Cell Physiol Biochem 2018;45:1566-1580 \begin{tabular}{l|l|l|l} 
DOI: 10.1159/000487693 & O 2018 The Author(s). Published by S. Karger AG, Basel \\
www.karger.com/cpb
\end{tabular}

cells (Fig. 2E), while clonogenic survival was significantly increased by upregulation of LINC00707 expression (Fig. 2F). Similarity, 5-ethynyl-2-deoxyuridine EdU(red)/DAPI (blue) immunostaining confirmed that downregulation of LINC00707 remarkably weakened proliferation capacity, while its overexpression had an inverse effect (Fig. 2G and H). Taken together, these findings suggest that LINC00707 has oncogenic characteristics and participates in LAD cell proliferation.

\section{Downregulation of LINC00707 induces G1 arrest and apoptosis of LAD cells}

The cell cycle and apoptosis are considered as key factors regulating cell growth. Thus, flow-cytometric analysis was performed to evaluate these factors. As shown in Fig. 3A and 3B, SPCA1 and A549 cells transfected with si-LINC00707 1\# and 3\# showed cell-cycle arrest at the G1-G0 phase compared to cells transfected with scrambled control. Furthermore, qRTPCR and western blot assays revealed that the mRNA and protein levels of several cell cyclerelated genes were greatly suppressed in cells transfected with LINC00707 siRNAs (Fig. 3C and D), demonstrating that LINC00707 is involved in cell-cycle regulation. Fig. 3E shows that the proportion of apoptotic cells treated with si-LINC00707 1\# and 3\# was remarkably increased in LAD cells. These results confirmed that the proliferation decrease of LAD cells transfected with LINC00707 siRNAs may be attributable to the induction of G1 arrest and apoptosis.

\section{Knockdown of LINC00707 expression inhibits LAD cell migration in vitro and cell metastasis} in vivo

To further evaluate whether LINC00707 contributes to cell migration, transwell assays were performed. As shown in Fig. 4A, the migratory ability of SPCA1 and A549 cells was inhibited by LINC00707 knockdown. In contrast, upregulation of LINC00707 improved the migration capacity of LAD cells (Fig. 4B). We next investigated whether LINC00707 knockdown impairs cell metastasis in vivo by establishing a mouse model of lung metastasis. SPCA1 cells stably transfected with sh-LINC00707 or empty vector were injected into the tail veins of nude mice. Five weeks after injection, the group with LINC00707 downregulation exhibited reduced numbers of metastasis nodules compared to those in the control (Fig. 4C

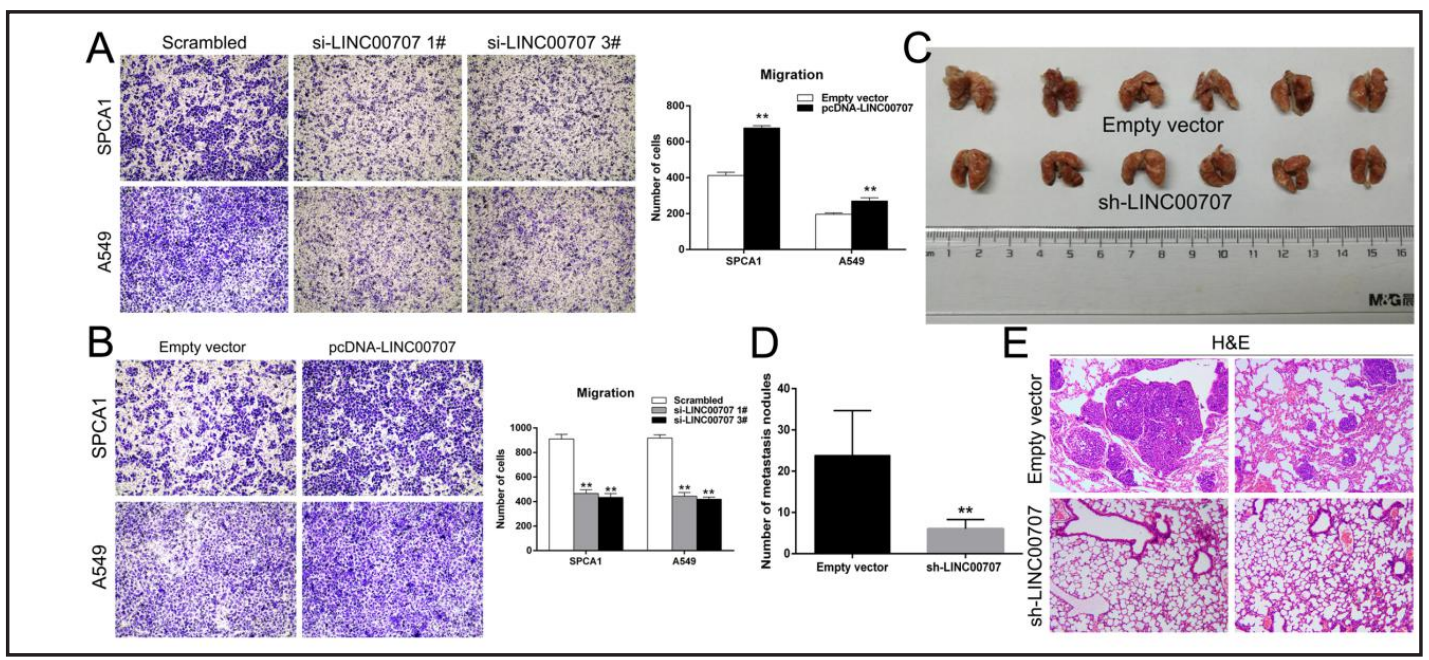

Fig. 4. Knockdown of LINC00707 expression inhibits LAD cell migration in vitro and cell metastasis in vivo. (A) Transwell assays were performed to determine migration in LAD cells transfected with si-LINC00707 1\#, 3\# or scrambled. (B) Migration ability was investigated by transwell assays in LAD cells transfected with pcDNA-LINC00707 or empty vector. (C) Lungs from mice in each experimental group. (D) Numbers of metastasis nodules on the lung surfaces were counted. (E) Visualization of the entire lung, and H\&E stained lung sections. Values are shown as the mean \pm s.d in three independent experiments. ${ }^{*} \mathrm{P}<0.05,{ }^{*} \mathrm{P}<0.01$. 
and D). This difference was further verified by hematoxylin and eosin (H\&E) staining of lung sections (Fig. 4E). The results manifested that LINC00707 may act as an oncogene that can promote the migration and metastasis of LAD cells in vitro and in vivo, respectively.

\section{LINC00707 promotes tumorigenesis of LAD cells in vivo}

A xenograft tumor model was constructed to further evaluate the tumor-promoting effect of LINC00707 and its impact on the progression of LAD in vivo. A549 cells stably transfected with sh-LINC00707 or empty vector were inoculated into nude mice. Xenograft tumors appeared at the injection site of all mice. Twenty-eight days after injection, it is smaller tumors that mice in sh-LINC00707 group developed than those in the control group (Fig. 5A and B). Thus, LINC00707 knockdown decreased tumor cell proliferation, as tumor growth in the sh-LINC00707 group was substantially slower than that in the empty vector group (Fig. 5C). Furthermore, the sh-LINC00707 group showed lower tumor weights than the control group did (Fig. 5D). QRT-PCR analysis also revealed that tumor tissues in the shLINC00707 group expressed lower LINC00707 levels than those of the control group (Fig. 5E). Ki-67 levels reflect proliferation ability. Immunohistochemical (IHC) analysis revealed that Ki-67 staining displayed lower intensity in tumor tissues from the sh-LINC00707 group compared to those from the control (Fig. 5F). Generally, these results show that LINC00707 has oncogenic properties in LAD in vivo.

\section{Cdc42 is a downstream target gene regulated by LINC00707}

To identify potential targets regulated by LINC00707, we performed highsolution microarray to detect changes in the gene expression profiles of SPCA1 cells in which LINC00707 expression was suppressed (Fig. 6A). SPCA1 cells were transfected with scrambled siRNA or siLINC00707 3\# for $48 \mathrm{~h}$. Analysis revealed 562 differentially expressed genes (fold-change $>2$, $\mathrm{p}<0.05), 133$ of which were upregulated in LINC00707 knockdown cells and 399 showing decreased expression (see online suppl. material, Table S2). Data collected from the Gene Ontology (GO) database was used to explore the underlying pathways activated by LINC00707. The prominent GO categories were related to apoptosis, differentiation, and cell

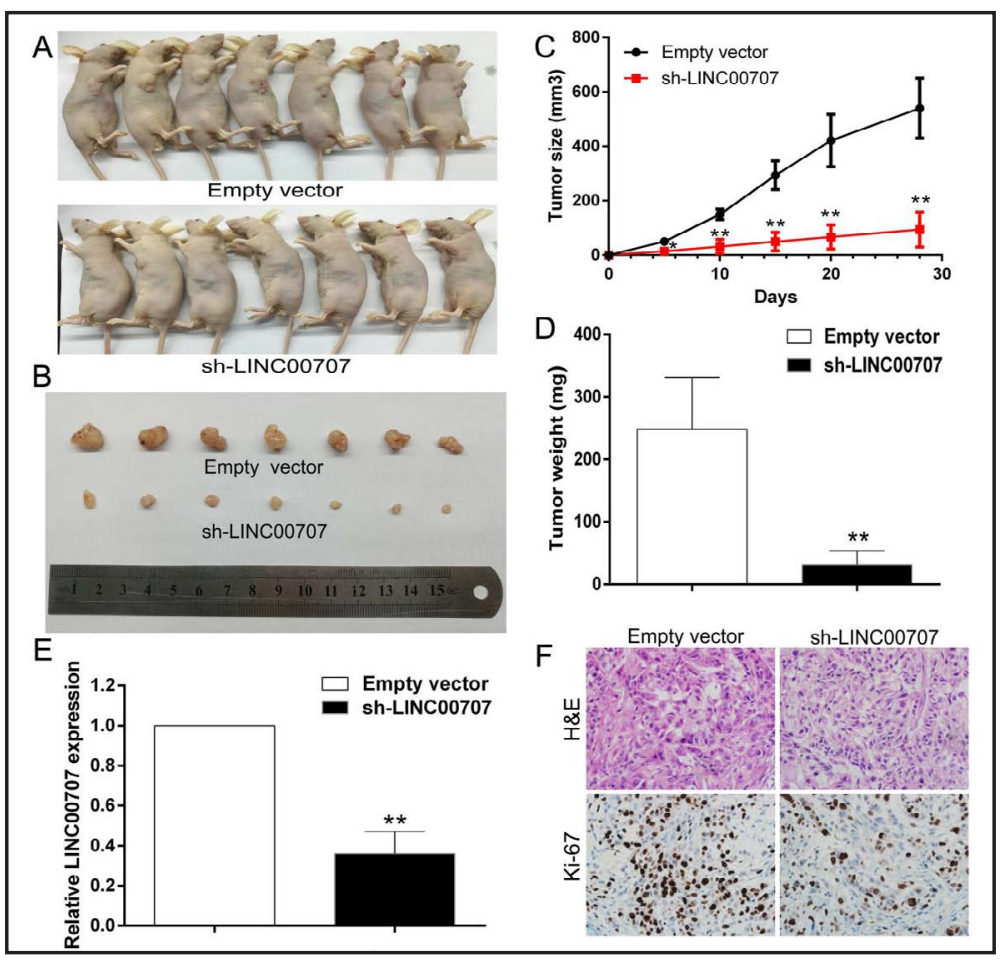

Fig. 5. LINC00707 knockdown inhibits tumorigenesis of LAD cells in vivo. (A and B) Empty vector or sh-LINC00707 was transfected into A549 cells, which were injected in the BALB/c-nude mice $(n=7)$, respectively. Tumors before and after carrying from the nude mice. (C)Tumor volumes were calculated after injection every five days. Points, mean $(n=6)$; bars indicate SD.(D) Tumor weights were represented as means of tumor weights \pm SD. (E) QRT-PCR was used to detect the average expression of LINC00707 in xenograft tumors $(\mathrm{n}=7)$. ( $\mathrm{F}$ ) The tumor sections were under H\&E staining and IHC staining using antibodies against ki-67. ${ }^{*} \mathrm{P}<0.05,{ }^{* *} \mathrm{P}<0.01$. 
Fig. 6. Cdc42 is a downstream target gene regulated by LINC00707. (A) Meancentered, hierarchical clustering of 562 transcripts altered in scrambled siRNA-treated cells and si-LINC00707-treated cells, with three repeats. (B and C) QRT-PCR analysis in si-LINC00707-treated or pcDNA- LINC00707treated LAD cells showed altered mRNA level of genes involved in cell apoptosis and migration upon LINC00707 depletion. Values are shown as the mean \pm s.d in three independent experiments. ${ }^{*} \mathrm{P}<$ $0.05,{ }^{* *} \mathrm{P}<0.01$.

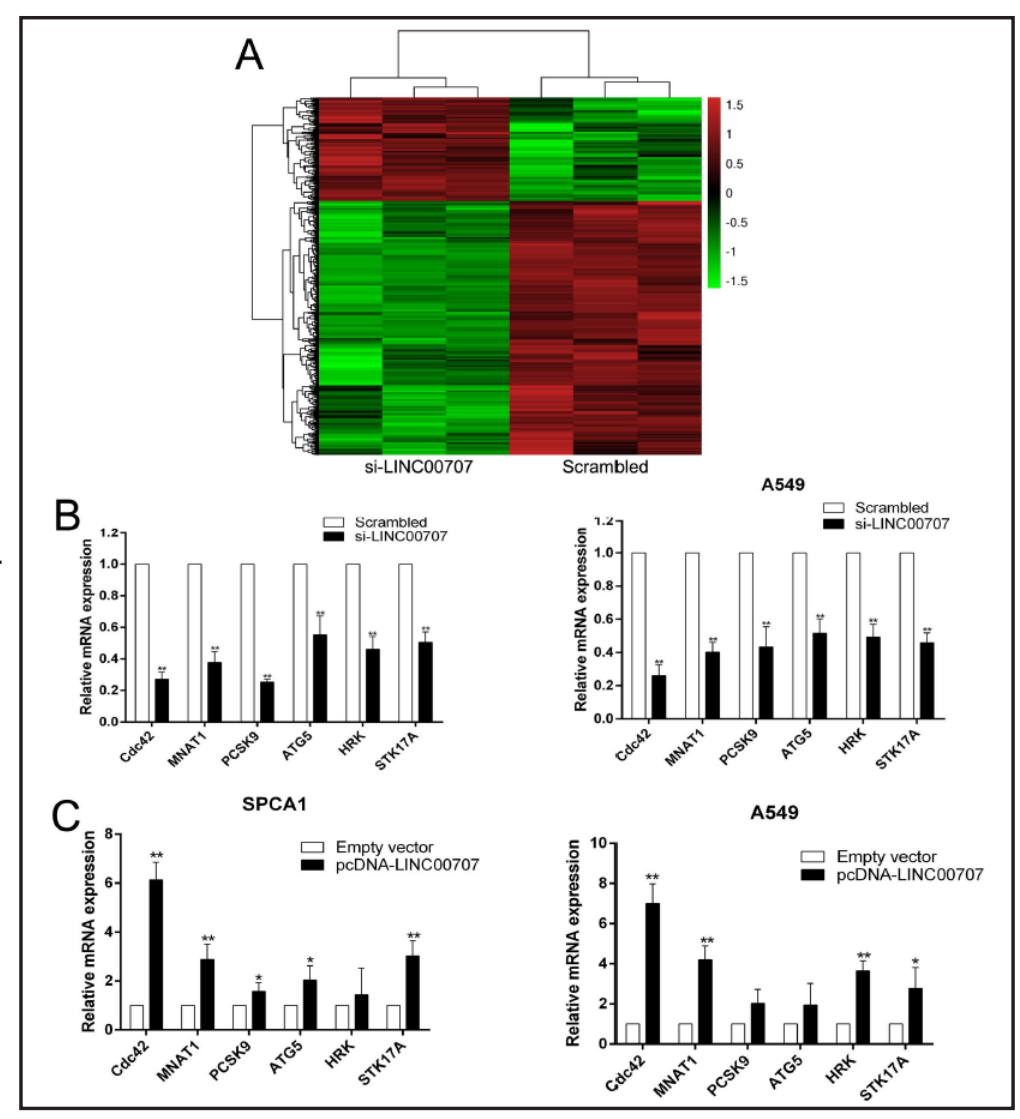

migration. The GO results were in accordance with our experimental findings. Because our study focused on lncRNA-regulated tumorigenesis, which is characterized by rapid proliferation and metastasis, subsequent experiments mainly evaluated these aspects.

To further identify the target gene regulated by LINC00707, qRT-PCR assays were performed to verify the changes in several differentially expressed mRNAs involved in apoptosis, migration, and growth pathways based on the results of microarray and GO analyses. As shown in Fig. 6B, after LINC00707 knockdown, Cdc42, MNAT1, PCSK9, ATG5, HRK, and STK17A showed decreased expression. These data are consistent with the microarray results in general. Additionally, LINC00707 overexpression showed inverse results (Fig. 6C). According to our data, Cdc42 was the most significantly downregulated mRNA in LINC00707-depleted LAD cells. These results suggest that Cdc42 is an important downstream gene of LINC00707.

\section{Cdc42 is oncogenic and potential involvement in the function of LINC00707}

To assess the role of Cdc42 in LAD, we detected the expression level of Cdc42 by qRTPCR in 64 pairs LAD and corresponding normal tissues. The expression level of Cdc42 was distinctly increased in the $64 \mathrm{LAD}$ tissues compared to that in adjacent normal tissues (Fig. 7A). QRT-PCR was also conducted in the LAD cell lines and normal bronchial epithelium cells. The results showed similar results to those in tissues (Fig. 7B). Next, we synthesized siRNA and shRNA for Cdc42 for functional assays in SPCA1 and A549 cells and the expression of Cdc42 was effectively inhibited (Fig. 7C and (see online suppl. material) Fig. S1B). Next, MTT and colony formation assays were performed to evaluate cell viability. Our findings revealed that downregulation of Cdc42 impaired cell proliferation (Fig. 7D and E). Flow- cytometric analysis also showed that Cdc42 downregulation induced G1 arrest and apoptosis in LAD cells (Fig. 7F and G). Subsequently, transwell assays indicated that knockdown of Cdc42 inhibited cell migration (Fig. 7H). These results demonstrated downregulation of Cdc42

\section{KARGER}


Fig. 7. Downregulation of Cdc42 inhibits LAD cells proliferation and is involved in the oncogene function of LINC00707. (A) Relative expression of $\mathrm{Cdc} 42$ in LAD tissues $(\mathrm{n}=64)$ and the paired noncancerous tissues ( $\mathrm{n}=64$ ) analyzed by qRT-PCR and normalized to GAPDH. (B) QRT-PCR assay examined Cdc42 expression in normal bronchial epithelial cell line (16HBE) and LAD cell lines. (C) QRT-PCR analysis of $\mathrm{Cdc} 42$ expression in LAD cells transfected with control (scrambled) and si-Cdc42. (D) MTT assays were performed to determine viability of LAD cells treated with the control and si-Cdc42. (E) Colony formation assays were used to detect the proliferation of sh-Cdc42transfected LAD cells. Colonies were counted and captured. (F and G) Cell cycle and apoptosis of LAD cells was investigated by flow-cytometry. (H) Transwell assays were conducted to evaluate migration of LAD cells. (I) Analysis of the relationship between Cdc42 and LINC00707 expression. (J) Colony formation assays were used to determine the proliferation for sh-Cdc42 and pcDNA-LINC00707 cotransfected LAD cells. (K) MTT

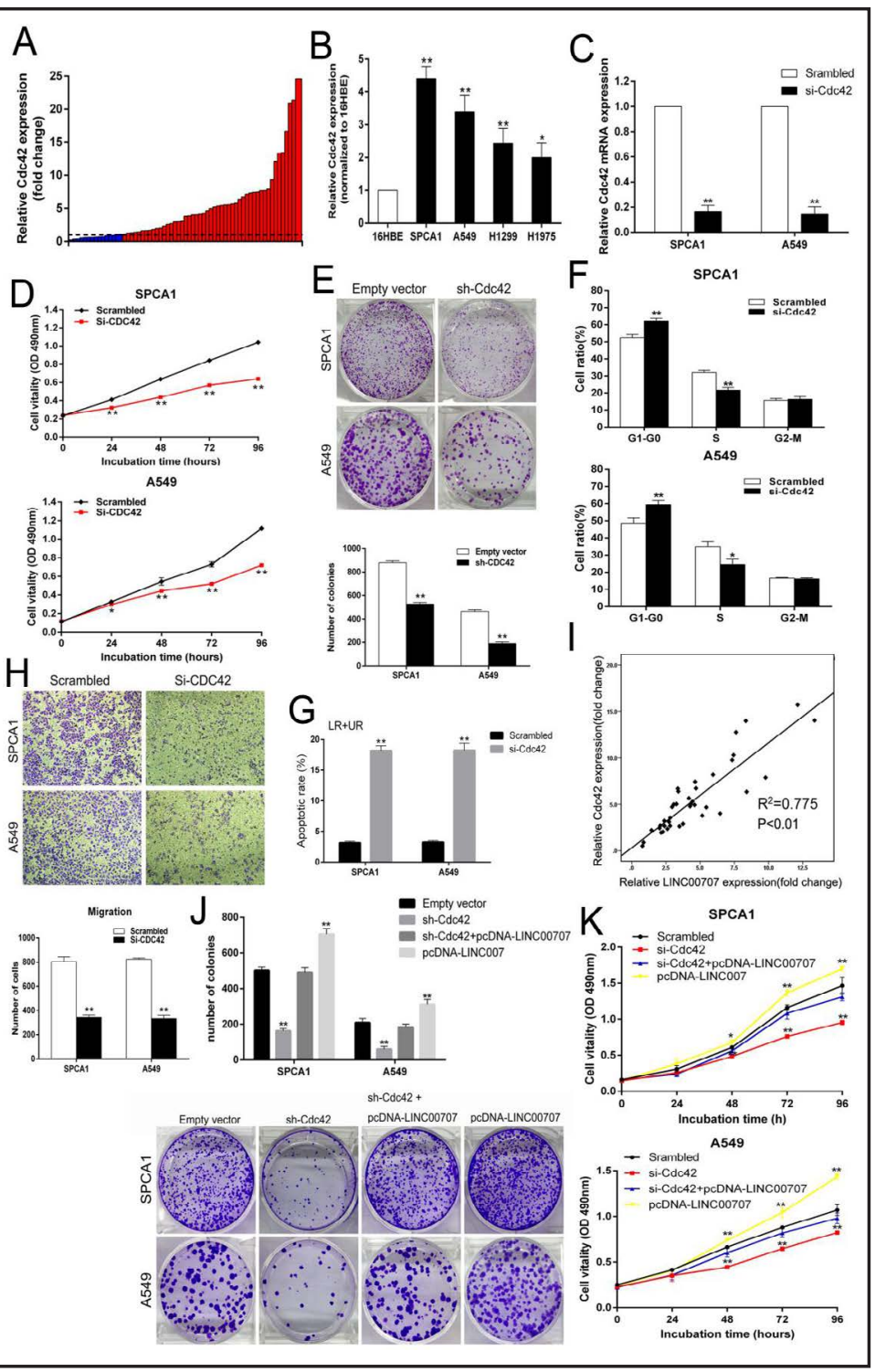
assays were used to determine the cell viability for si-Cdc42 and pcDNA-LINC00707 co-transfected LAD cells. Values are shown as the mean \pm s.d in three independent experiments. ${ }^{*} \mathrm{P}<0.05,{ }^{* *} \mathrm{P}<0.01$.

inhibited the proliferation and migration of LAD cells, similar to the results of LINC00707 knockdown.

Furthermore, the expression level of Cdc42 was positively correlated with the LINC00707 expression level in LAD tissues (Fig. 7I). Accordingly, rescue assays were performed to investigate whether Cdc42 is involved in the LINC00707-mediated promotion of cell growth in LAD. SPCA1 and A549 cells were co-transfected with pcDNA-LINC00707 and si-Cdc42. MTT and colony formation experiments showed that Cdc42 interference partially rescued pcDNA-LINC00707-induced proliferation in SPCA1 and A549 cells (Fig. 7J and K). In summary, these findings suggest that LINC00707 promotes cell proliferation in part by activating the expression of Cdc42 in LAD. 


\section{Discussion}

Over the past several decades, next-generation sequencing and high-resolution microarray have greatly advanced cancer research. Recent studies have revealed that expression alterations of lncRNAs are among the driving forces of tumorigenesis. Our previous study identified a pseudogene-derived IncRNA, DUXAP8, that promotes cell proliferation and migration by associating with PRC2 to epigenetically silence PLEKHO1 expression in gastric cancer (GC) [20]. Moreover, IncRNA HOXA-AS2 promotes GC cell proliferation via epigenetically inhibiting P21/PLK3/DDIT3 [21]. In addition, LINC00673 regulates cell proliferation, metastasis, and epithelial-mesenchymal transition (EMT) by sponging miR-150-5p in NSCLC [22]. However, studies of the relationship between IncRNAs and LAD are limited. Therefore, the identification of cancer-related lncRNAs, particularly in LAD, may reveal the link between the oncogene and tumor suppressor network.

In this study, we evaluated the function and potential mechanisms of LINC00707 in LAD. Our data showed that the expression level of LINC00707 in LAD tissues was significantly higher than in corresponding tissues, and LINC00707 upregulation was correlated with advanced malignant clinical characteristics. Gain or loss of function assays were performed to explore the effect of LINC00707 on LAD. The date elaborated that LINC00707 promoted cell proliferation by regulating cell cycle and apoptosis both in vitro and in vivo. Cell migration was also improved by LINC00707 overexpression. These results revealed the carcinogenic effects of LINC00707 in LAD.

Through functional assays, we explored the mechanism of LINC00707 activity. Subsequently, differentially expressed genes were screened by microarray analysis in cells transfected with scrambled and si-LINC00707, respectively. We identified 562 genes (foldchange $>2, \mathrm{p}<0.05$ ) showing differential expression. Enrichment analyses revealed that these genes are generally involved in biological processes related to cell adhesion, junction, metastasis and apoptosis. QRT-PCR assays identified Cdc42 as a target gene of LINC00707.

Cell division cycle 42 (Cdc42) belongs to the Rho GTPases family and Ras superfamily [23]. Cdc42 was initially identified as a mediator of cell polarity and division in Saccharomyces cerevisiae [24,25]. Recent studies show that deregulation of Cdc42 is involved in the activation of many cellular cascades such as cell polarity, cytoskeleton remolding, proliferation, migration, adhesion membrane trafficking, and transportation, and leads to the development of many pathological disorders including cancers in humans [26-28]. Stimulation of the G-protein-coupled receptor kinase interacting protein 1 (GIT1)-Rac/Cdc42 axis is important for cell motility in NSCLC, and thus activating of Rac1/Cdc42 is critical for the GIT1-induced invasiveness of NSCLC [29]. Proliferation of lung cancer cells was suppressed by upregulation of miR-137 both in vivo and in vitro by regulating the expression of Cdc42 and Cdk6 to some extent [30]. Interferon regulatory factor 4 binding protein activates Rac1, RhoA, and Cdc42 to regulate EMT and motility in breast cancer cells [31]. It has been shown that Cdc42 serve as an oncogene in various tumors such as breast cancer, colorectal cancer, and ovarian cancer [32-34]. These findings suggest that Cdc42 plays an oncogenic role in cancers by influencing cell metastasis and proliferation. Our experiments confirmed the oncogenic contribution of Cdc42. Inhibition of Cdc42 led to attenuation of growth and migration in LAD cells. A rescue assay manifested that Cdc42 was potentially involved in the function of LINC00707. In summary, LINC00707 was correlated with its target Cdc42 and thus promoted the proliferation and migration of LAD cells.

It is well-known that lncRNAs are involved in a myraid of molecular functions in numerous processes [11]. LncRNAs use various mechanisms to execute their functions, such as epigenetic, transcriptional, and post-transcriptional regulation as well as regulation of microRNAs (miRNAs) [12]. Our data also showed that LINC00707 is mainly located in the cytoplasm (see online suppl. material, Fig. S1C). LncRNAs located in the cytoplasm have been implicated in post-transcriptional regulatory events, partially by interacting with miRNAs and Argonaute 2 (Ago2) [35, 36]. Numerous reports have described the intricate interplay between IncRNAs and miRNAs [37]. LncRNA-PXN-AS1 containing exon 4 protects PXN 


\section{Cellular Physiology Cell Physiol Biochem 2018:45:1566-1580 \begin{tabular}{ll|l} 
DOI: 10.1159/000487693 & $\begin{array}{l}\text { O 2018 The Author(s). Published by S. Karger AG, Basel } \\
\text { www.karger.com/cpb }\end{array}$ \\
\cline { 2 - 3 }
\end{tabular} \\ Ma et al.: Oncogenic Functions of LINC00707 in LAD}

mRNA against degradation induced by the miR-24-Ago2 complex, thereby increasing PXN expression in hepatocellular carcinoma [38]. LINC00673 sponges miR-150-5p and regulates ZEB1 expression indirectly via a competing endogenous RNAs (ceRNA) mechanism in NSCLC [22]. Moreover, IncRNAs function as ceRNAs or miRNA sponges, representing an extensive form of gene expression regulation at the post-transcriptional level [37, 39]. Furthermore, miRNAs together with the Argonaute family, with Ago 2 as the core component, serve as components of ribonucleoprotein complexes or RNA-induced silencing complexes to function post-transcriptionally in the cytoplasm $[40,41]$. The SNHG5/miR-32/KLF4 axis functions as an important regulator of GC cell proliferation and migration, and the expression of SNHG5 is suppressed by miR-32 in an Ago2-dependent manner [42]. HOTAIR acts as a ceRNA, sponging the expression of miR-331-3p by binding to Ago2, thereby regulating the derepression of HER2 post-transcriptionally in GC [43]. The interaction probabilities of LINC00707 and Ago2 were predicted using bioinformatic tool (http://pridb.gdcb.iastate. edu/RPISeq/). The results supported the correlation between LINC00707 and Ago2, with an RF classifier and SVM classifier of 0.8 and 0.98 , respectively ((see online suppl. material, Fig. S1D). However, identification of both miRNAs paired with LINC00707 and the combination of LINC00707 and Ago2 requires further analysis.

\section{Conclusion}

Taken together, our results revealed that LINC00707 was overexpressed in LAD tissues and its upregulation may be related to several clinicopathological indices. The capacity of LINC00707 to promote LAD cell proliferation and migration may be achieved partially through the interaction with Cdc42 expression. Generally, our study provides a novel perspective for the mechanism by which LINC00707 regulates LAD cell growth and migration. Therefore, LINC00707 may be a new biomarker for diagnosis and latent therapy target of LAD. However, the other possible approaches related to the participation of LINC00707 in LAD biological processes require further investigation.

\section{Acknowledgements}

We thank Professor Wei De (Department of Biochemistry and Molecular Biology, Nanjing Medical University) for the help of experiments. This project was supported by grants from National Natural Science Foundation of China (No. 81773109 and No. 81702340), National Scientific Foundation of Jiangsu Province of China (BK20151582), the Fund of the priority Academic Program Development of Jiangsu Higher Education Institution (JX10231801) and the National key clinical specialist construction Programs of China (2014).

\section{Disclosure Statement}

The authors declare no conflicts of interest.

\section{References}

1 Torre LA, Bray F, Siegel RL, Ferlay J, Lortet-Tieulent J, Jemal A: Global cancer statistics, 2012 CA Cancer J Clin 2015; 65:87-108.

-2 Liu J, Wan L, Lu K, Sun M, Pan X, Zhang P, Lu B, Liu G, Wang Z: The Long Noncoding RNA MEG3 Contributes to Cisplatin Resistance of Human Lung Adenocarcinoma. PLoS One 2015; 10:e0114586.

-3 Siegelin MD, Borczuk AC: Epidermal growth factor receptor mutations in lung adenocarcinoma. Lab Invest 2014; 94:129-137. 


\section{Cellular Physiology Cell Physiol Biochem 2018;45:1566-1580 \begin{tabular}{l|l|l|l} 
DOI: 10.1159/000487693 & O 2018 The Author(s). Published by S. Karger AG, Basel \\
www.karger.com/cpb
\end{tabular} \\ Ma et al.: Oncogenic Functions of LINC00707 in LAD}

4 da Cunha Santos G, Shepherd FA, Tsao MS: EGFR mutations and lung cancer. Annu Rev Pathol 2011; 6:4969.

5 Li Z, Yamada S, Wu Y, Wang KY, Liu YP, Uramoto H, Kohno K, Sasaguri Y: Polypeptide $\mathrm{N}$-acetylgalactosaminyltransferase- 6 expression independently predicts poor overall survival in patients with lung adenocarcinoma after curative resection. Oncotarget 2016; 7:54463-54473.

6 Mohni KN, Kavanaugh GM, Cortez D: ATR pathway inhibition is synthetically lethal in cancer cells with ERCC1 deficiency. Cancer Res 2014; 74:2835-2845.

-7 Xu TP, Liu XX, Xia R, Yin L, Kong R, Chen WM, Huang MD, Shu YQ: SP1-induced upregulation of the long noncoding RNA TINCR regulates cell proliferation and apoptosis by affecting KLF2 mRNA stability in gastric cancer. Oncogene 2015; 34:5648-5661.

8 Djebali S, Davis CA, Merkel A, Dobin A, Lassmann T, Mortazavi A, Tanzer A, Lagarde J, Lin W, Schlesinger F, Xue C, Marinov GK, Khatun J, Williams BA, Zaleski C, Rozowsky J, Roder M, Kokocinski F, Abdelhamid RF, Alioto T, et al.: Landscape of transcription in human cells. Nature 2012; 489:101-108.

- Consortium EP: An integrated encyclopedia of DNA elements in the human genome. Nature 2012; 489:5774.

10 Harrow J, Frankish A, Gonzalez JM, Tapanari E, Diekhans M, Kokocinski F, Aken BL, Barrell D, Zadissa A, Searle S, Barnes I, Bignell A, Boychenko V, Hunt T, Kay M, Mukherjee G, Rajan J, Despacio-Reyes G, Saunders G, Steward C, et al.: GENCODE: the reference human genome annotation for The ENCODE Project. Genome Res 2012; 22:1760-1774.

11 Wilusz JE, Sunwoo H, Spector DL: Long noncoding RNAs: functional surprises from the RNA world. Genes Dev 2009; 23:1494-1504.

12 Li X, Wu Z, Fu X, Han W: IncRNAs: insights into their function and mechanics in underlying disorders. Mutat Res Rev Mutat Res 2014; 762:1-21.

13 Huarte M: The emerging role of IncRNAs in cancer. Nat Med 2015; 21:1253-1261.

14 Liao J, Yu X, Hu X, Fan J, Wang J, Zhang Z, Zhao C, Zeng Z, Shu Y, Zhang R, Yan S, Li Y, Zhang W, Cui J, Ma C, Li L, Yu Y, Wu T, Wu X, Lei J, et al.: IncRNA H19 mediates BMP9-induced osteogenic differentiation of mesenchymal stem cells (MSCs) through Notch signaling. Oncotarget 2017; 8:53581-53601.

15 Magistri M, Velmeshev D, Makhmutova M, Faghihi MA: Transcriptomics Profiling of Alzheimer's Disease Reveal Neurovascular Defects, Altered Amyloid-beta Homeostasis, and Deregulated Expression of Long Noncoding RNAs. J Alzheimers Dis 2015; 48:647-665.

16 Chen QN, Chen X, Chen ZY, Nie FQ, Wei CC, Ma HW, Wan L, Yan S, Ren SN, Wang ZX: Long intergenic noncoding RNA 00152 promotes lung adenocarcinoma proliferation via interacting with EZH2 and repressing IL24 expression. Mol Cancer 2017; 16:17.

17 Chen J, Zhang K, Song H, Wang R, Chu X, Chen L: Long noncoding RNA CCAT1 acts as an oncogene and promotes chemoresistance in docetaxel-resistant lung adenocarcinoma cells. Oncotarget 2016; 7:6247462489.

18 Sun J, Pan LM, Chen LB, Wang Y: LncRNA XIST promotes human lung adenocarcinoma cells to cisplatin resistance via let-7i/BAG-1 axis. Cell Cycle 2017; 16:2100-2107.

19 Liao WT, Wang X, Xu LH, Kong QL, Yu CP, Li MZ, Shi L, Zeng MS, Song LB: Centromere protein H is a novel prognostic marker for human nonsmall cell lung cancer progression and overall patient survival. Cancer 2009; 115:1507-1517.

20 Ma HW, Xie M, Sun M, Chen TY, Jin RR, Ma TS, Chen QN, Zhang EB, He XZ, De W, Zhang ZH: The pseudogene derived long noncoding RNA DUXAP8 promotes gastric cancer cell proliferation and migration via epigenetically silencing PLEKHO1 expression. Oncotarget 2017; 8:52211-52224.

21 Xie M, Sun M, Zhu YN, Xia R, Liu YW, Ding J, Ma HW, He XZ, Zhang ZH, Liu ZJ, Liu XH, De W: Long noncoding RNA HOXA-AS2 promotes gastric cancer proliferation by epigenetically silencing P21/PLK3/DDIT3 expression. Oncotarget 2015; 6:33587-33601.

22 Lu W, Zhang H, Niu Y, Wu Y, Sun W, Li H, Kong J, Ding K, Shen HM, Wu H, Xia D, Wu Y: Long non-coding RNA linc00673 regulated non-small cell lung cancer proliferation, migration, invasion and epithelial mesenchymal transition by sponging miR-150-5p. Mol Cancer 2017; 16:118.

23 Machesky LM, Hall A: Rho: a connection between membrane receptor signalling and the cytoskeleton. Trends Cell Biol 1996; 6:304-310.

24 Johnson DI, Pringle JR: Molecular characterization of CDC42, a Saccharomyces cerevisiae gene involved in the development of cell polarity. J Cell Biol 1990; 111:143-152. 


\section{Cellular Physiology Cell Physiol Biochem 2018;45:1566-1580

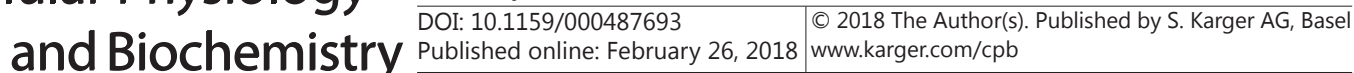 \\ Published online: February 26, 2018 wh.karg}

-25 Adams AE, Johnson DI, Longnecker RM, Sloat BF, Pringle JR: CDC42 and CDC43, two additional genes involved in budding and the establishment of cell polarity in the yeast Saccharomyces cerevisiae. J Cell Biol 1990; 111:131-142.

26 Qadir MI, Parveen A, Ali M: Cdc42: Role in Cancer Management. Chem Biol Drug Des 2015; 86:432-439.

27 Baschieri F, Confalonieri S, Bertalot G, Di Fiore PP, Dietmaier W, Leist M, Crespo P, Macara IG, Farhan H: Spatial control of Cdc42 signalling by a GM130-RasGRF complex regulates polarity and tumorigenesis. Nat Commun 2014; 5:4839.

-28 Reymond N, Im JH, Garg R, Vega FM, Borda d'Agua B, Riou P, Cox S, Valderrama F, Muschel RJ, Ridley AJ: Cdc42 promotes transendothelial migration of cancer cells through beta1 integrin. J Cell Biol 2012; 199:653-668.

29 Chang JS, Su CY, Yu WH, Lee WJ, Liu YP, Lai TC, Jan YH, Yang YF, Shen CN, Shew JY, Lu J, Yang CJ, Huang MS, Lu PJ, Lin YF, Kuo ML, Hua KT, Hsiao M: GIT1 promotes lung cancer cell metastasis through modulating Rac1/Cdc42 activity and is associated with poor prognosis. Oncotarget 2015; 6:36278-36291.

30 Zhu X, Li Y, Shen H, Li H, Long L, Hui L, Xu W: miR-137 inhibits the proliferation of lung cancer cells by targeting Cdc42 and Cdk6. FEBS Lett 2013; 587:73-81.

31 Zhang Z, Yang M, Chen R, Su W, Li P, Chen S, Chen Z, Chen A, Li S, Hu C: IBP regulates epithelial-tomesenchymal transition and the motility of breast cancer cells via Rac1, RhoA and Cdc42 signaling pathways. Oncogene 2014; 33:3374-3382.

-32 Hirsch DS, Shen Y, Wu WJ: Growth and motility inhibition of breast cancer cells by epidermal growth factor receptor degradation is correlated with inactivation of Cdc42 Cancer Res 2006; 66:3523-3530.

-33 Sakamori R, Yu S, Zhang X, Hoffman A, Sun J, Das S, Vedula P, Li G, Fu J, Walker F, Yang CS, Yi Z, Hsu W, Yu DH, Shen L, Rodriguez AJ, Taketo MM, Bonder EM, Verzi MP, Gao N: CDC42 inhibition suppresses progression of incipient intestinal tumors. Cancer Res 2014; 74:5480-5492.

-34 Guo Y, Kenney SR, Muller CY, Adams S, Rutledge T, Romero E, Murray-Krezan C, Prekeris R, Sklar LA, Hudson LG, Wandinger-Ness A: R-Ketorolac Targets Cdc42 and Rac1 and Alters Ovarian Cancer Cell Behaviors Critical for Invasion and Metastasis. Mol Cancer Ther 2015; 14:2215-2227.

-35 Zhang Z, Zhu Z, Watabe K, Zhang X, Bai C, Xu M, Wu F, Mo YY: Negative regulation of IncRNA GAS5 by miR21. Cell Death Differ 2013; 20:1558-1568.

-36 Staff PG: Correction: fMiRNA-192 and miRNA-204 Directly Suppress lncRNA HOTTIP and Interrupt GLS1Mediated Glutaminolysis in Hepatocellular Carcinoma. PLoS Genet 2016; 12:e1005825.

37 Tay Y, Rinn J, Pandolfi PP: The multilayered complexity of ceRNA crosstalk and competition. Nature 2014; 505:344-352.

38 Yuan JH, Liu XN, Wang TT, Pan W, Tao QF, Zhou WP, Wang F, Sun SH: The MBNL3 splicing factor promotes hepatocellular carcinoma by increasing PXN expression through the alternative splicing of IncRNA-PXNAS1. Nat Cell Biol 2017; 19:820-832.

39 Qi X, Zhang DH, Wu N, Xiao JH, Wang X, Ma W: ceRNA in cancer: possible functions and clinical implications. J Med Genet 2015; 52:710-718.

40 Filipowicz W, Bhattacharyya SN, Sonenberg N: Mechanisms of post-transcriptional regulation by microRNAs: are the answers in sight? Nat Rev Genet 2008; 9:102-114.

-41 Izaurralde E: Elucidating the temporal order of silencing. EMBO Rep 2012; 13:662-663.

-42 Zhao L, Han T, Li Y, Sun J, Zhang S, Liu Y, Shan B, Zheng D, Shi J: The lncRNA SNHG5/miR-32 axis regulates gastric cancer cell proliferation and migration by targeting KLF4. FASEB J 2017; 31:893-903.

-43 Liu XH, Sun M, Nie FQ, Ge YB, Zhang EB, Yin DD, Kong R, Xia R, Lu KH, Li JH, De W, Wang KM, Wang ZX: Lnc RNA HOTAIR functions as a competing endogenous RNA to regulate HER2 expression by sponging miR331-3p in gastric cancer. Mol Cancer 2014; 13:92. 